\title{
Characteristics of flow rate, head loss and basket pressure drop relationship in a Rocla Versa Trap (VT) stormwater pollutant trap (SPT) in a 3-D laboratory scale model
}

\author{
H. K. Saberi \& H. Nikraz \\ Department of Civil Engineering, School of Engineering, \\ Curtin University of Technology, Western Australia
}

\begin{abstract}
Rapid development of the big cities and suburbs around the world in the last century has created a situation in which the main focus of urban rainwater runoff is the disposal of the stormwater as quickly and efficiently as possible to the disposal site, without considering the water quality at outfall. This has contributed to the decline of water quality of the rivers, lakes and other receiving bodies. Whilst some progress has been made towards the reduction in pollution at the source, it is the non-point sources of pollution entering the water runoff system at various points and from different sources that is the most difficult to monitor and manage.

One of the basic and at the same time major concepts in recent years is to capture and treat as much of the pollutant as possible which is discharged to the drainage system at source or possibly close to the source as the first step. One of the most popular and economical approaches is the use of an off-line stormwater pollutant trap (SPT).

Recent developments in stormwater quality management have seen the introduction of SPTs, which are generally the most up-to-date devices designed to capture and store gross pollutants and some micro-pollutants, for subsequent removal and disposal.

In this experiment a scale model of a Versa Trap series, a gross pollutant trap that utilises an upstream diversion weir pit to divert the Design Treatment Flow (DTF) into a cylindrical treatment chamber which houses a stainless steel basket, is considered. Treated flow passing through the basket screen is returned via the exit chamber to the diversion pit downstream of the weir, re-entering the drainage system. Peak flows in excess of the DTF bypass the diversion pit over the weir into the pipeline downstream.

A mathematical relationship between the flow rate and head loss across the basket can be established for the device, which allows the designer to specify a suitable weir height in the diversion pit and to clarify the allowable range for removing and cleaning the basket at its optimum maintenance situation.

Keywords: runoff, stormwater, receiving bodies, off line, pollutant trap, gross pollutant, treatment chamber, diversion pit, weir, head loss.
\end{abstract}




\section{Introduction}

We should dramatically change our view about the way we think and talk about the resources of our freshwater - mainly rainwater or stormwater. "Every time we think about stormwater management, let's think about drinking water management instead. Every time we talk about managing stormwater, let's talk about managing drinking water. Today we treat rainwater as if it were a misbehaving student: we send it to detention until we can figure out how to get it away from us permanently" Shore [1]. By definition, any rain that falls on roofs or collects on paved areas such as roads, driveways, or footpaths is called stormwater. The stormwater drainage system is different and separate from the sewerage system. The stormwater that flows over the land picks up all kinds of impurities and chemicals that are toxic to the natural environment. Even naturally occurring nutrients, which are not poisonous in small concentrations, may be carried by flows in such great quantities and/or concentrations that the natural ecosystems cannot cope. This type of pollutant can eventually lead to toxic algal blooms and other pollution problem in our waterways.

The majority of pollutant material monitored in Australia was vegetation (typically 70 percent by mass) with plastics and paper discarded from pedestrian and motorist activities making up most of the remainder (Alison [2]).

Stormwater pollutants includes three major parts:

Organic materials such as leaves, grass trimmings and sediments

Chemicals such as motor oil, coolant, grease, detergents, fertiliser and paint

Litter such as bags and cigarette butts (Parliament of the Commonwealth of Australia [3]).

The management of runoff stormwater can be divided into two basic types of best management practices (BMP): preventive measures (non-structural practices) and control measures (structural practices). Preventive measures include removing debris, minimising pollutants, diverting runoff, containing stormwater and educating employees, while control measures may include dry and wet detention basins, infiltration devices, oil and grease traps and constructed wetlands (Kreuzer [4]).

This paper presents an independent prototype study in a controlled measure of an off-line stormwater pollutant trap (SPT) using a scale model to provide a relationship between head loss and pollutant removal efficiency in the offline SPT system. The motivation behind this experiment is to establish a logical and reliable result for the rapid production of these devices in the marketplace, often with little or no independent evaluation of their performance.

Historical pollutant removal effectiveness studies of BMPs have consisted of on field monitoring installations under a wide range of pollutant loadings, storm events, monitoring procedures, and product sizes which have led to obvious limitations on pollutant loading and repeatability of measured results (AlHamdani et al. [5]). Therefore any testing experiment that is based on rainfall with aforementioned data will be handicapped by these limitations. 
The purpose of testing stormwater pollutant traps with controlled conditions is to provide accurate and repeatable documentation of the performance of specific structural best management practice that can be used to predict its performance in a variety of field conditions. Controlled testing allows us to isolate specific parameters that affect the pollutant removal efficiencies which are difficult, if not impossible, to isolate in the field. These parameters include: the flow rate, the influent concentration, and the percentage of concentration.

\section{Treatment mechanisms in the scaled model}

Stormwater quality and stormwater quantity controls are not commonly exclusive and in many situations the control solutions specified for water quantity control provide significant water quality benefit as well. The stormwater quality control can be achieved by a variety of stormwater storage and sedimentation technique. The performance requirements of the stormwater pollutant traps (SPTs) storage vary in proportion to a number of site specific conditions including catchment type, anticipated pollutants, runoff flow, capture and entrapment objectives, groundwater level, ground water, soil permeability and classification of receiving water, among others.

The pollutant removal efficiency of the SPT - one of the major issues considered in the design, selection, and installation of this type of trap - is affected to some extent by the degree to which the screen is blocked. There are two ways to accomplish this, either through flow reduction and/or an increase in basin surface area. The necessary modification to the existing sediment storage basin may be cost prohibitive, or physical constrains such as space limitation, which can prevent implementation. This also affects the head loss across the SPT, and needs to be taken into account in the establishment of the flow/head loss and flow/weir height relationship formulae.

The design and construction of the scale model was based on the existing Versa Trap series A, an off-line gross pollutant trap, which is similar to many basins in the region.

So the resulting basin dimension data used to design experimental parameters for scale model construction and testing.

In this research we tested the scale model under a $0 \%$ blocked screen, and then increased it to $22 \%$ blockage and after that adding $10 \%$ increment each time until we reached a $77 \%$ total blockage of the screen. The main reason for limiting the state of blockage to $77 \%$ in this research is that the published performance data for the VTA is stated as applying to a 50\% screen blocked condition, and it is recommended that cleaning be carried out at or before this stage is reached. The actual data that was used, however, is as measured in the laboratory at $75 \%$ blocked, which provides a reasonable factor of safety.

The Versa Trap SPT utilises three distinct processes in the separation of pollutants from contaminated water:

1) The vortex phenomenon encourages solids to move inwards to the low velocity region at the centre of the cylindrical treatment chamber, where they 
settle to the sediment sump or rise to the surface. The vortex is generated by tangential introduction of the influent into the cylindrical chamber above the screen.

2) The stainless steel screen captures all suspended particles of size greater than screen aperture size. The aperture size is generally $5 \mathrm{~mm}$, by common definition of a gross pollutant, but may be varied to suit the characteristics of gross pollutants generated by a particular catchment.

3) Fine sediment passing through the screen will settle (depending on size and specific gravity) in the exit chamber, where vertical velocities determine the capture performance.

The inlet pipe to the storm pollutant trap is connected to the inner cylinder of the trap (which is called the active or treatment chamber) at a tangent, creating a vortex inside the basket. The runoff water carrying gross pollutants passes through the stainless steel basket, which is inserted inside the treatment chamber, leaving pollutants trapped in the basket. Treated water then passes out of the SPT via the outer cylinder (which is also called the external or exit chamber) as shown in Figure 1.

The test flow created in the laboratory model was a base-mounted centrifugal pump capable of circulating up to $12 \mathrm{l} / \mathrm{s}$ of flow in the piping network.

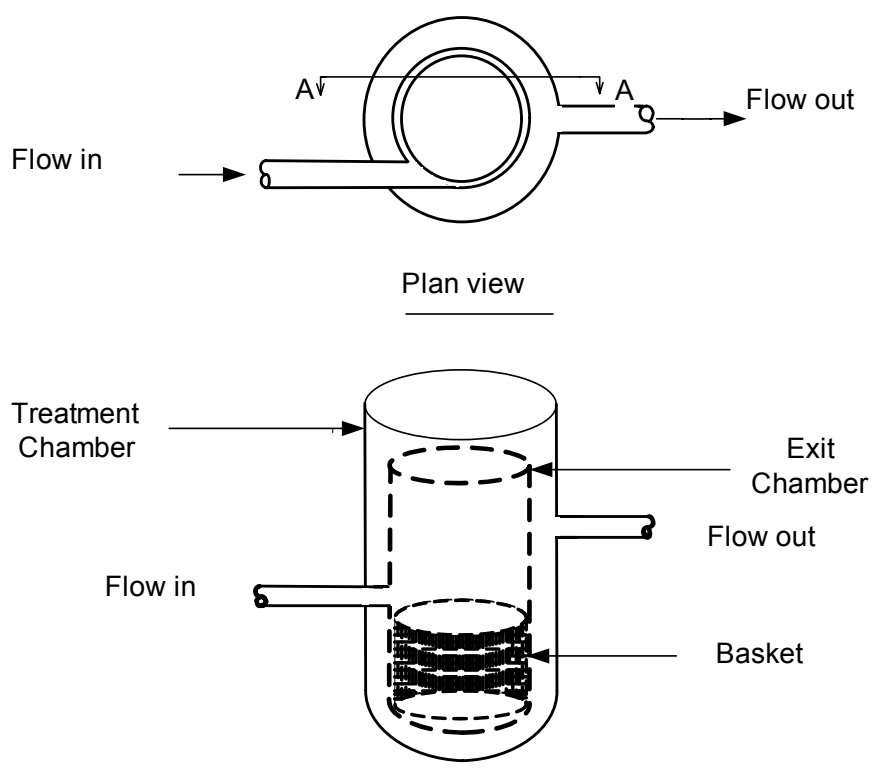

Section A-A

Figure 1: $\quad$ Stormwater pollutant trap (SPT). 


\section{Methods and materials}

A series of tests with different screen blockage and flow rates were conducted in the open laboratory environment of the Civil Engineering Department of the Curtin University of Technology in Western Australia with the aforementioned pump connected to the reservoir which is equipped with a $90^{\circ} \mathrm{V}$-notch and an attached hook gage weir box measuring the water level above the v-notch. The pump and reservoir connected to a scaled SPT model network via a valve and PVC pipes, tees, and elbows, returning the circulated water to the reservoir. Two piezometer tubes were installed on the pipe at section 1 and 2 (Chow [6]), as shown in figure 2.

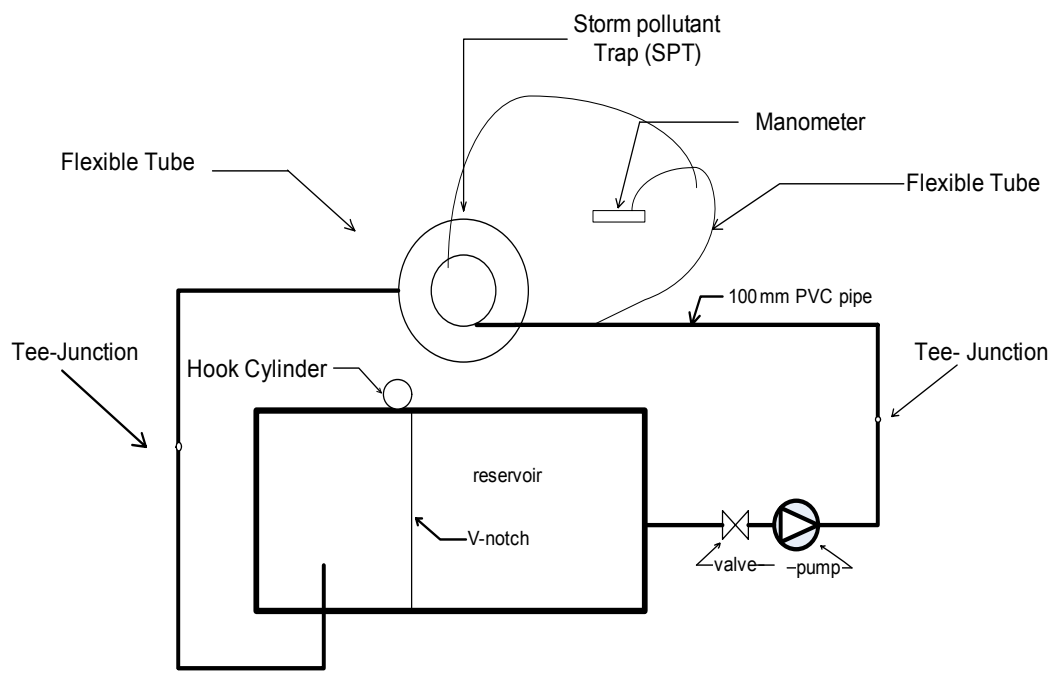

Figure 2: Off-line SPT laboratory network piping plan.

When depths are shallow and as a result the velocities are low for a reliable current-meter measurement of discharge, a V-notch weir is particularly suitable because of its sensitivity at low flows.

For a circular pipe carrying stormwater and running part-full, the following formulas for flow rate, angle, and area of the pipe are used:

$$
\begin{aligned}
& Q=\frac{8}{15} C \tan \frac{\theta}{2} \sqrt{2 g_{c} h^{5}} \\
& \alpha=\cos ^{-1}\left(\alpha-\frac{y}{r}\right) \\
& A=r^{2}\left(\alpha-\frac{\sin 2 \alpha}{2}\right)
\end{aligned}
$$

where: 
$Q$ is the flow rate in $\mathrm{m}^{3} / \mathrm{sec}$.

$h$ is the water level over weir's notch in meters

$\theta$ is triangular - notch weir angle

$g_{c}$ is gravity $9.81 \mathrm{~m} / \mathrm{sec}^{2}$

$\alpha$ is the angle in radian

$y$ is the height of water in pipe in meter

$r$ is the radius of pipe in meter

$C$ is the coefficient of discharge

$A$ is the area of pipe in $m^{2}$ [7]

In this experiment a stainless steel $90^{\circ} \mathrm{V}$-notch was selected and attached to the $1000 \mathrm{~mm}$ wide $\left(\mathrm{L}_{\mathrm{c}}\right)$ reservoir with maximum $\mathrm{h}$ of $156 \mathrm{~mm}$ and considering all requirements of the $\mathrm{V}$-notch weir (ASME [7]). Also a hook gauge weir box was assembled and attached to the reservoir with the pointer reading zero when tip of the hook is at the level of notch apex as shown in figure 3.

$\mathrm{L}_{\mathrm{c}}=$ Width of the approach channel

$\mathrm{Lh}=$ Width of the distance $\mathrm{h}$ above the apex

$\mathrm{HI}=$ approach depth of water to the weir

$P=$ elevation of the weir above bottom of the

channel

$h=$ Height of fluid flow over the weir

$\Theta=$ Angle of triangular notched weir when

the tip of the hook is at level of notch apex
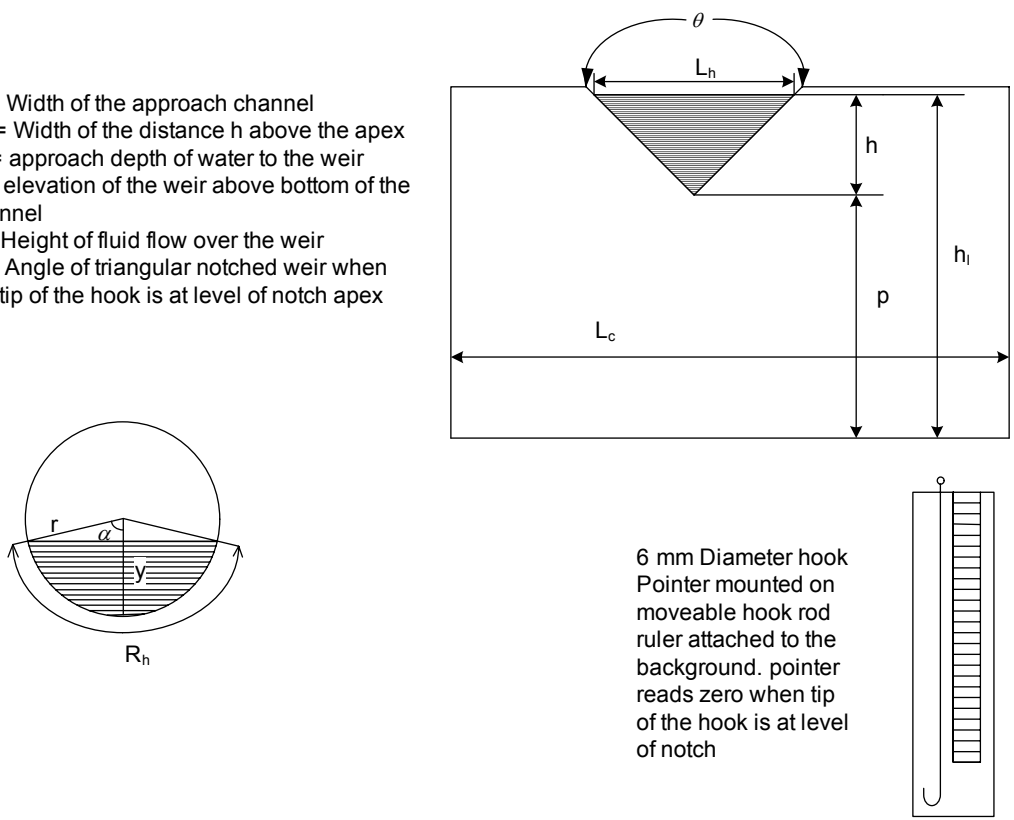

Figure 3: $\quad$-notch and hook gauge weir box.

The value of the coefficient, $\mathrm{C}$, to be used in the equation (1) for a V-notch weir is mainly dependent on the notch angle, $\theta$, and only slightly on the head, $\mathrm{h}$, as shown in figure 3 above (ASME [7]).

The scaled SPT model was built based on the drawing with $100 \mathrm{~mm}$ PVC pipe for network piping and assembled using PVC.U Pipe Cement as shown in figure 4 , below. 
The reservoir was filled up to the apex of the weir with water and the zero point of the hook gauge weir was calibrated for an accurate reading for each set of experiments. The flow rates were adjusted by opening the valve of the network, which was located after the pump a fraction of turn each time. Flow rates were determined using the vee notch weir. The head loss across the model was determined by comparing the heights of the water in the inlet and outlet manometers. Results were tabulated and charted to establish the mathematical relationship between head loss and flow rate. This process was repeated several times for each percent of screen blockage.

There are mainly three ways to calculate the head loss in the pipe, a) HazenWilliams equation, equation 4, b) Manning's equation, equation 5, and c) DarcyWeisbach equation, equation (6) (White [8]).

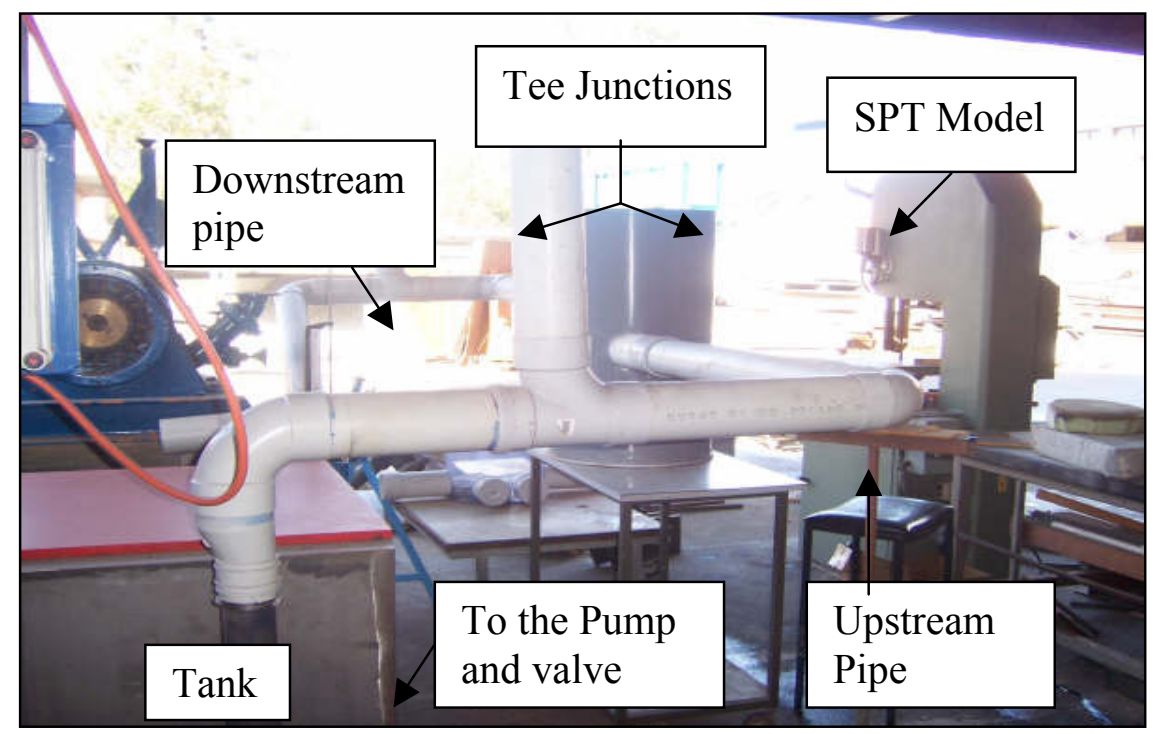

Figure 4: $\quad$ Scaled SPT experimental setup.

$$
\begin{gathered}
H_{L}=\frac{10.74 L}{D^{2.63}\left(\frac{Q}{C}\right)^{1.85}} \quad \text { Hazen-Williams equation } \\
H_{L}=\frac{5.29 L(n Q)^{2}}{D^{16 / 3}} \quad \text { Manning's equation } \\
H_{L}=f \frac{L}{D} \frac{V^{2}}{2 g}
\end{gathered}
$$

where:

$f$ is the dimensionless friction factor

$v$ is the velocity

$g$ is the acceleration due to gravity 
$D$ is the pipe diameter

$L$ is the pipe length

$h_{L}$ is the head loss over pipe length, L.

$Q$ is the flow rate

$C$ is the Hazen-Williams roughness coefficient

$n$ is the Manning roughness coefficient

The Hazen-Williams and the Manning equations are both empirical and have dimensional coefficients that are affected by the roughness of conduit. The Darcy-Weisbach equation provides a more rational basis for flow computations. The friction factor, $f$, varies with the Reynolds Number of the flow and the relative roughness of the pipe except for fully rough turbulent flow where $f$ is independent of the Reynolds Number (Keller et al [9]).

Since the manometers were connected to the network close to the SPT, the friction loss of the short length of the network's pipe run of the system was consequently negligible and therefore not considered, but the friction of the entrance and exit and blocked basket had been considered as a whole system in this experiment.

\section{Results and discussion}

By considering the fact that the outlet pipe is $143 \mathrm{~mm}$ above the inlet pipe, therefore all static head measurements were taken from the outlet invert level. The recorded data was tabulated and analysed and 44\% up to $66 \%$ blockage were amongst the best situated data, which the combined curve illustrates in Figure 5.

The head loss across the weir pit is an important consideration when designing a pipeline.

The inlet head for each percentage of the screen is plotted in the graph below and it can be seen that the blocked screen in the range of $44 \%$ to $77 \%$ bear in a very close relation to each other with the $\mathrm{R}^{2}$ (quadratic regression) value of over 0.99 implying that by using second degree polynomial

$$
\mathrm{h}_{\mathrm{L}(\mathrm{i})}=-1.7 \mathrm{Q}^{2}+30 \mathrm{Q}+258
$$

where $\mathrm{h}_{\mathrm{L}(\mathrm{i})}$ is inlet head loss in millimetre, one can estimate the entrance head loss by entering a desired value of flow rate (Q) in litre per second in the above equation.

For the outlet flow each increment of flow data has been measured and plotted versus the screen percentage blocked and the final graph is based on $44 \%$ to $77 \%$ of the blocked basket with a quadratic regression value of 0.99 which indicates that the outlet head loss for the SPT can be estimated by using the second degree.

Polynomial of the form,

$$
\mathrm{h}_{\mathrm{L}(\mathrm{e})}=-1.7 \mathrm{Q}^{2}+19.5 \mathrm{Q}+251
$$

where $\mathrm{h}_{\mathrm{L}(\mathrm{e})}$ is the outlet or exit head loss in millimetre and flow rate $(\mathrm{Q})$ is in litre per second. The graph for the combination of $44 \%$ to $77 \%$ (average of $60 \%$ ) blocked outlet head loss is shown in Figure 6. 


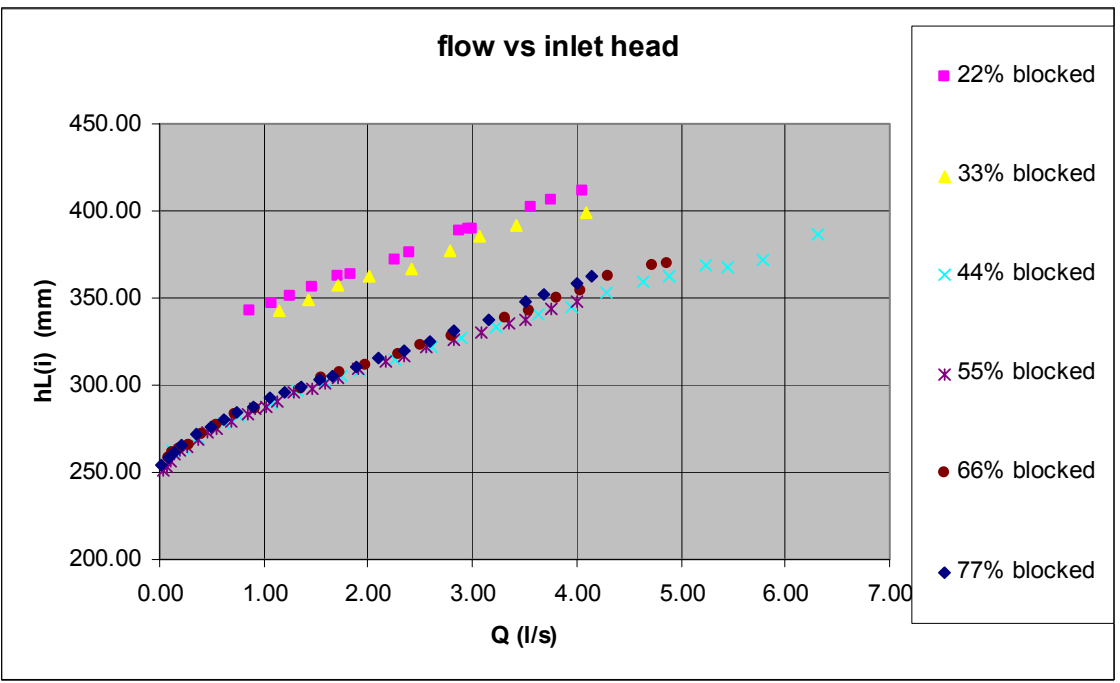

Figure 5: $\quad$ Flow vs. inlet head.

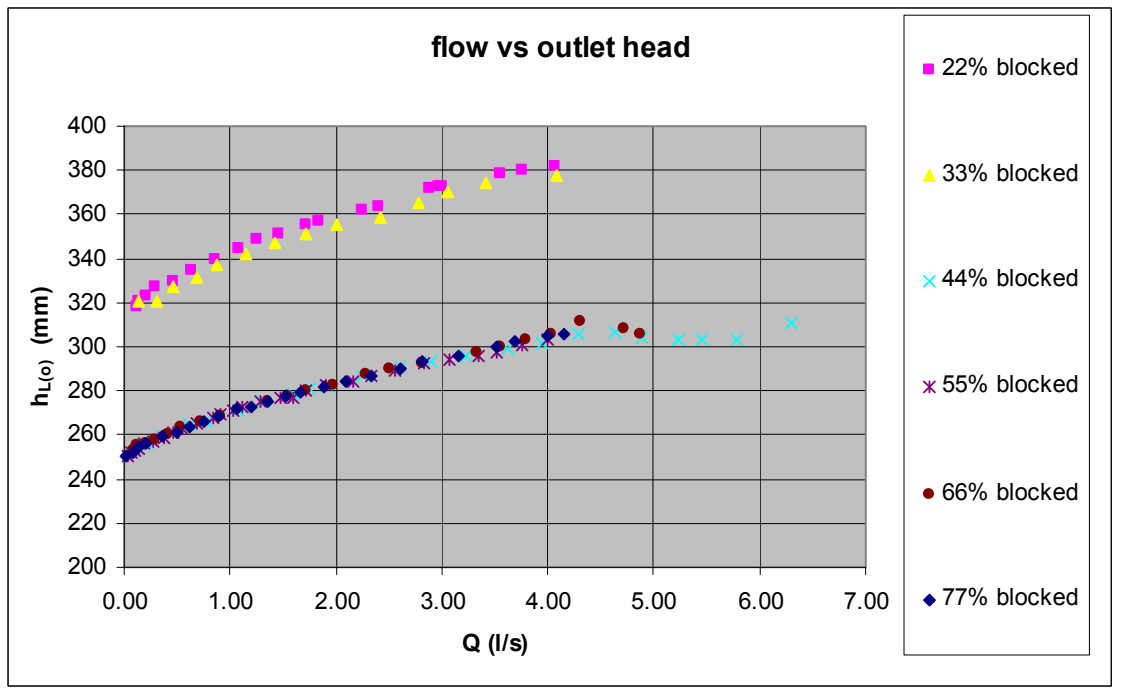

Figure 6: Flow vs. outlet head.

By knowing the total head values (static and velocity) of entering and exit water at a wide range of the flow rates and screen blockages, the total head losses for the flow in the SPT were obtained as shown in Figure 7.

Since the values of the screen blockage from zero up to $33 \%$ are in the lower range of the total pressure drop and are less significant compared to $44 \%-77 \%$ screen blockage, which are totally in different grouping, they are in an acceptable 


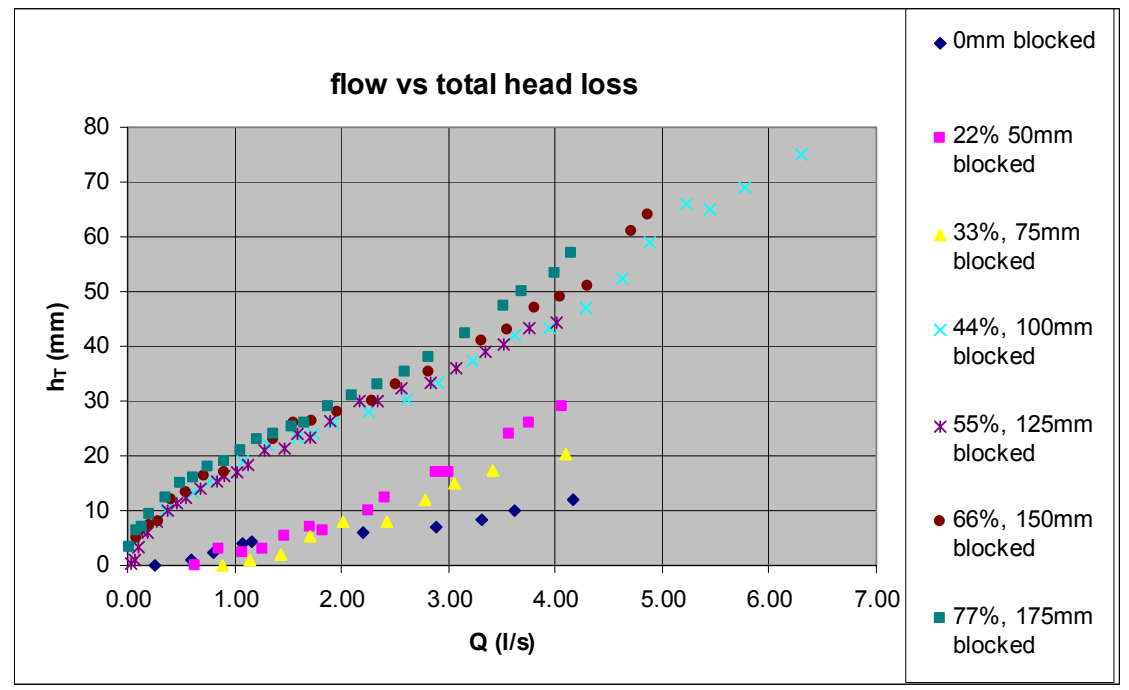

Figure 7: $\quad$ Flow vs. total head loss for $44 \%-77 \%$ blocked basket.

range of the total pressure drop head loss and flow rate. So the final equation that we come up with is from the plotted combination curve of $44 \%-77 \%$ values, with a mean square value of 0.97 . Therefore graph 3 can be used for estimating the total head loss of any size of SPT of this specific type (Versa Trap series A) with combined data from 100 to $170 \mathrm{~mm}$ (44\% to $77 \%$ ) screen blocked or using the following equation

$$
\mathrm{h}_{\mathrm{T}}=-0.006 \mathrm{Q}^{2}+10.8 \mathrm{Q}+6.2
$$

By substituting the flow rate value (Q) in litre per second in above equation, one can get the total head loss of the main prototype very accurately.

Now for the set of acceptable range of the data $(44 \%-77 \%)$, by subtracting the inlet and outlet effects of the pipe mainly the head loss due to the sudden expansion in entrance to the inner chamber and sudden contraction to the exit pipe during the outflow, the result will be the net pressure drop due to the basket blockage. This is shown in the Figure 8.

The resultant logarithmic equation, which is concluded from the above graph with a regression value of 0.91 , is;

$$
\mathrm{h}_{\mathrm{L}(\mathrm{B})}=6.64 \mathrm{Ln}(\mathrm{Q})+19.3
$$

Since the experiment was conducted using a geometrically and dynamically similar scaled model, the relationships represented by the formulae hold good for the full size units.

\section{Conclusion}

This stage of the research project i.e. establishing a relationship between flow rate and basket pressure drop across a VTA off-line pollutant trap - has been 


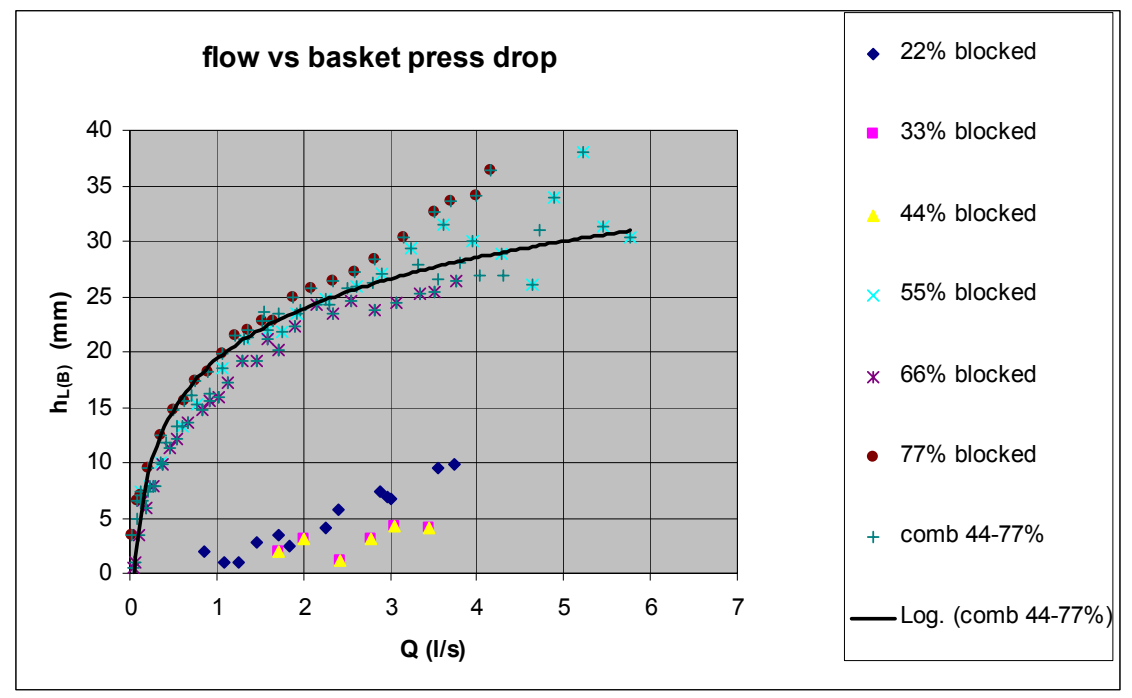

Figure 8: $\quad$ Flow vs. basket pressure drop 44\%-77\% blocked.

satisfactorily achieved. This relationship depicted in Figure 8 is formulated in equation (11) below;

$$
\mathrm{H}_{(\mathrm{TB})}=6.64 \operatorname{Ln}(\mathrm{Q})+19.3
$$

The above equation provides a mathematical model which satisfies the correlation criteria to a level of 0.91 , and can be used with confidence in the establishment of the relationship between flows, head loss and basket pressure drop in a off - line VTA SPT.

Therefore by increasing the basket's surface area, or in other words increasing the off-line trap capacity, the capacity of the trap to hold more debris will increase.

Provided relative dimension are retained (included inlet and outlet pipes) at an acceptable range of created pressure drop and flow rate, the appropriate SPT could be selected.

\section{References}

[1] Shore, D., Stormwater Management. cleanerriver.blogspot.com, 2007. 11(1).

[2] Alison, R.A., Innovate technology reduces stormwater trash. ProQuest Science Journal, 1999. 130(2): p. 28.

[3] Parliament of the Commonwealth of Australia, The Value of Water: Inquiry into Australia's management of urban water, C. Senate Environment, Editor. 2006, The Parliament of the Commonwealth of Australia

[4] Kreuzer, H., Planning for a rainy day: Best management practices for controlling industry stormwater runoff Pollution Engineering, 2001. 33(2): p. 6 . 
[5] Al-Hamdani, et al., Performance reconnaissance of stormwater proprietary best management practices. Engineering Science and Health Part A, 2007. 42: p. 10.

[6] Chow, V.T., Open-Channel Hydraulics. (1956,reissued 1988), New York: McGraw-Hill.

[7] ASME, Fluid Meters Their Theory and Application. sixth ed. 1971, New York, N.Y.: The American Society of Mechanical Engineers, ASME. 113117.

[8] White, F.M., Fluid Mechanics. Fifth Edition ed. 2003, New York: McGrawHill

[9] Keller, et al., Comparison of Discharge From Alternative Pipeline Material. 1995. 